\title{
Path Plan of Unmanned Underwater Vehicle Using Particle Swarm Optimization
}

\author{
Sun Jian ${ }^{1}$, Liu Xing ${ }^{2, a}$ \\ ${ }^{1}$ Systems Engineering Research Institute, Beijing, China \\ ${ }^{2}$ Science and Technology on Underwater Acoustic Antagonizing Laboratory, Beijing, China \\ aliux.seri@163.com
}

Keywords: Unmanned Underwater Vehicle, Route Plan, Swarm Optimization

\begin{abstract}
This article proposes a method to solve route plan problem of unmanned underwater vehicle (UUV) using particle swarm optimization (PSO). Firstly, traditional electronic nautical map is preprocessed to decrease calculated amount in computation of route cost and improve plan instantaneity using technique of threaten circles covering. Secondly, choose appropriate route expression and cost computation. Lastly, route with minimum cost is received using particle swarm optimization. Result indicates that particle swarm optimization based on swarm intelligence can insure optimality most out of limited computation time, relative to traditional method using graph theory and dynamic programming.
\end{abstract}

\section{Introduction}

Path plan is the important content of task assignment of UUV, and purpose of path plan is to find the route from start point to goal point which satisfies UUV performance request. Generally, in the process of path plan, first step is to create a data structure represent path in the three dimension space, second step is to create appropriate cost function involve fuel, threat and etc, third step is to find optimum path using optimization algorithm which cost function achieves minimum.

Swarm Intelligence is popular method to solve path plan problem in the recent years, in which particle swarm algorithm is heuristic optimization method using heuristic random search. The algorithm is started from the swarm, every particle in the swarm represents solution to UUV path plan problem. In the search process, global optimum particle sharing global optimum information with every particle and local historical optimum particle sharing local optimum information with self particle. New position of particle is addition of global optimum position and local optimum position in some extent and after some iteration particle swarm finish search to global search space.

Differences of PSO and other swarm intelligence algorithm are: every particle has memory of local historical optimum solution and whole swarm has memory of global optimum solution. The principle of PSO is simple and parameters of PSO are less than other Swarm Intelligence algorithm to programming. So PSO is adapt to solve path plan problem has short time demand.

This article solves path plan problem of UUV, path plan method is constituted with four steps: (1) route representation; (2) cost function choose; (3) optimum path solution using PSO.

\section{Route representation}

Route representation is more important problem in the path plan, which affects cost value computation and effect of path plan. Better route representation not only represents motion character, but eliminates route not satisfies plan request from standby route, and increase efficiency of PSO algorithm. Traditional method of route representation is to plot grid map from the start to the goal, which is shown in the Picture (1). Using this method, Path plan problem can be seen as shortest route on the direct map with some weight. Limitation of this method is no matter search algorithm is powerful, method can not search path point between grid points and affect optimality of UUV plan route.

PSO algorithm is pointed to continue search space, and fit search map without grids, so take 
route representation as Picture (2). In the picture line between start and goal represents reference route, in the cruise of UUV, UUV positions should not be far from reference route. Line AB and CD represent plan boundary, in the progress of path plan, every route should not get across the $A B$ and CD boundaries. Sect equally line between start and goal and choose point on the sect line randomly as path point, we get prepared routes to PSO algorithm. As shown in Picture (2) grids of map is not existed so PSO can search points between grid points.

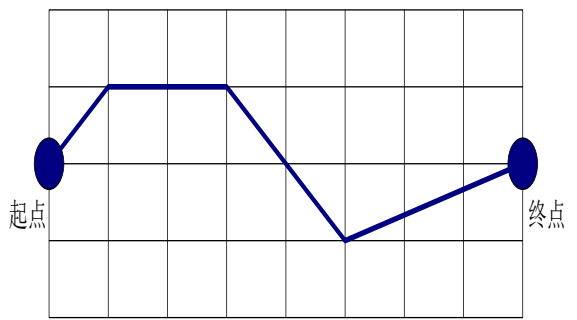

Picture (1): Traditional Route Representation

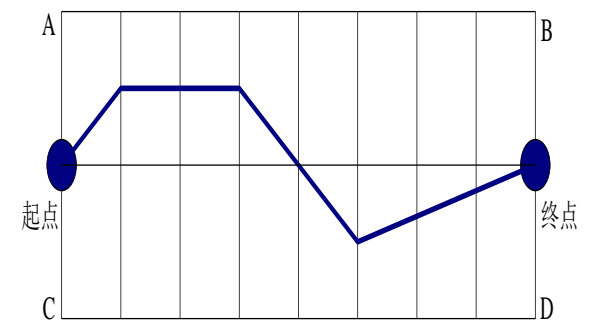

Picture (2): Route Representation of PSO Algorithm

\section{Cost Function}

Generally choose cruise distance, cruise duration, safety, smooth of route as factor of cost function to estimate UUV route equality. The route includes $\mathrm{n}$ points $p=\left[\left(x_{1}, y_{1}\right) \ldots\left(x_{i}, y_{i}\right) \ldots\left(x_{n}, y_{n}\right)\right]$ can be evaluated by following function

$$
\begin{aligned}
& f(p)=w_{d} \times \operatorname{dist}(p)+w_{c} \times \operatorname{clear}(p) \\
& +w_{s} \times \operatorname{smooth}(p)
\end{aligned}
$$

In which $w_{d} 、 w_{c}$ 和 $w_{s}$ is weights represent important degree of the terms, three factors of formula (1) represent cruise distance, safety and smooth respectively, in which:

$$
\operatorname{dist}(p)=\sum_{i=1}^{n-1} \sqrt{\left(x_{i+1}-x_{i}\right)+\left(y_{i+1}-y_{i}\right)}
$$

$\operatorname{dist}(p)$ is sum of distances of points on the route, $\operatorname{clear}(p)$ is punish to route which is crossed over threat circle, computation method is as following. Firstly, sect equally line between route point $\left(x_{i}, y_{i}\right)$ and route point $\left(x_{i+1}, y_{i+1}\right)$, and compute distance between sect point and center of threat circle, if the distance is bigger than radius of threat circle, sect point is safe, $\operatorname{clear}(p)=0$, otherwise sect point is not safe plus $\Delta$ to $\operatorname{clear}(p)$ as following:

$$
\operatorname{clear}(p)=\operatorname{clear}(p)+\Delta
$$

By traversal every point of route, we compute clear $(p)$ of the whole route.

$\operatorname{smooth}(p)$ represents smooth of the route and can be written as cosine sum of adjacent lines of route. Suppose points $\left(x_{i-1}, y_{i-1}\right),\left(x_{i}, y_{i}\right),\left(x_{i+1}, y_{i+1}\right)$ are continued points of the route, $d_{i-1, i}, d_{i, i+1}$, $d_{i-1, i+1}$ represent distances of $\left(x_{i-1}, y_{i-1}\right),\left(x_{i}, y_{i}\right) 、\left(x_{i}, y_{i}\right),\left(x_{i+1}, y_{i+1}\right) 、\left(x_{i-1}, y_{i-1}\right),\left(x_{i+1}, y_{i+1}\right)$, cosine value at point $\left(x_{i}, y_{i}\right)$ is as following:

$$
\gamma_{i}=180^{\circ}-a \cos \left(\frac{d_{i-1, i}^{2}+d_{i, i+1}{ }^{2}-d_{i-1, i+1}^{2}}{2 d_{i-1, i} d_{i, i+1}}\right)
$$

Calculation formula of $\operatorname{smooth}(p)$ is $\sum \gamma_{i}$.

\section{Optimal Route Using PSO Algorithm}

To solve optimal problem, solution of problem is particle of search space, every particle has position and velocity and cost function depended by route quality. The particle remembers and follows best particle of this generation, the particle renews position and velocity by two limit values: (1) Optimal solution of the particle self in iteration;(2) Optimal solution of whole particle swarm in 
the iteration. Standard PSO algorithm, velocity and position renew formulas are as follows:

$$
\left\{\begin{array}{l}
v_{i d}^{k+1}=\omega v_{i}^{k}+c_{1} r_{1}\left(p_{b e s t i d}^{k}-x_{i d}^{k}\right)+c_{2} r_{2}\left(g_{\text {bestid }}^{k}-x_{i d}^{k}\right) \\
x_{i d}^{k+1}=x_{i d}^{k}+v_{i d}^{k+1}
\end{array}\right.
$$

In the formula (5): $i \in N(1, m), m$ is number of particles of swarm; $d \in N(1, n), n$ is dimension of solution vector; $k$ is number of iteration; $c_{1}$ and $c_{2}$ are learning factors; $\omega$ is inertia weight; $r_{1}$ and $r_{2}$ are random numbers at [0,1].

Path Plan Steps are as follows:

Step1: Initialization, set learning factors $c_{1}$ and $c_{2}$, inertia weight $\omega$, generate randomly $m$ particles to form swarm in solution space. Positions of particle $x$ represent distance actual route away from reference route, generate randomly initial velocity of particles, maximum velocity is $30 \mathrm{~km}$;

Step 2: Compute pBest and gBest values according to formula (1);

Step 3: Renew particle velocity and position according to formula (5). To prevent particle to over boundaries, need saturation function to limit position of particles, as formula (6), in which range is $30 \mathrm{~km}$.

$$
x= \begin{cases}\text { range } & x>\text { range } \\ x & |x|<\text { range } \\ \text {-range } & x<- \text { range }\end{cases}
$$

Step 4: Estimate cost function of particles.

Step 5: Renew particle pBest according to cost function of the particle.

Step 6: Renew gBest according pBest of every particle.

Step 7: Check finish condition, if condition is satisfied, finish iteration, otherwise go to step 3. Finish condition is iteration of swarm achieve some value or difference of interval iteration is less than some error.

\section{Simulation Result}

Choose $(0,0)$ as start of route, $(100,100)$ as goal of route, generate swarm as Picture(3):

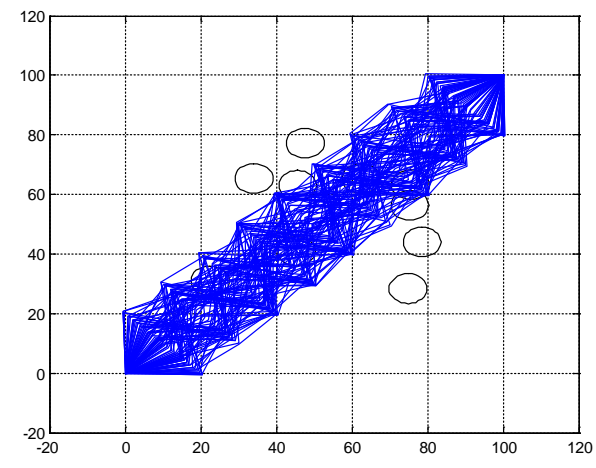

Picture (3): Initial Routes Represented By Initial Swarm

After 50 iterations routes represented by swarm is shown as Picture (4), it is obvious that swarm is converged and routes of UUV are consistent. Picture (5) is eventually optimal route of UUV. 


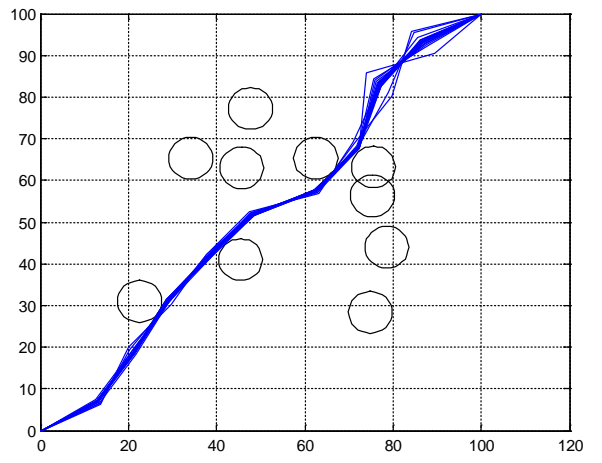

Picture (4): Routes Represented By Swarm After 50 Iterations

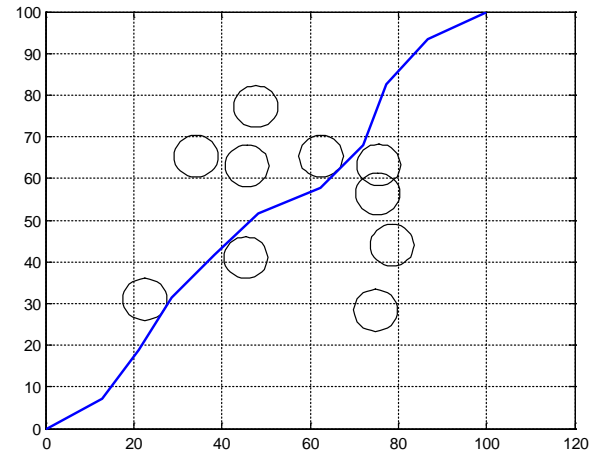

Picture (5): Optimal Route After 50 Iterations

\section{Conclusion}

PSO Algorithm has advantage that convergence speed is fast, algorithm is simple and program is easy, and paid to attention by scholar and engineer from appearance of PSO. At the same time, the algorithm not requires optimized function is differential and continued, so it quickly is applied in every optimized field. At present PSO algorithm is mainly applied in combined optimization and data mining.

This article is pointed to high dimension and nonlinear of path plan of UUV, apply PSO algorithm to plan process of UUV, propose steps to solve this kind of problems, simulate and compute optimal route. Simulated result shows validity of the algorithm.

\section{Reference}

[1] Gao Fang. Research on Intelligent Particle Swarm Optimization Algorithm[D].Harbin:Harbin University of Technology, 2008.06.

[2] Cao Jiangli .Researehon Key Teehno1ogy of AUV Path P1anning Issues [D]. Harbin:Harbin University of Technology, 2008.06.

[3] Wu Qin, Xu Xiaofei, Zhang jingwei, Ji Zhihong. Application of PSO Algorithm in Path Planning of Cruise Missile [J]. Ship Electr onic Engineering, 2010.11,Vol.30 No.11. 